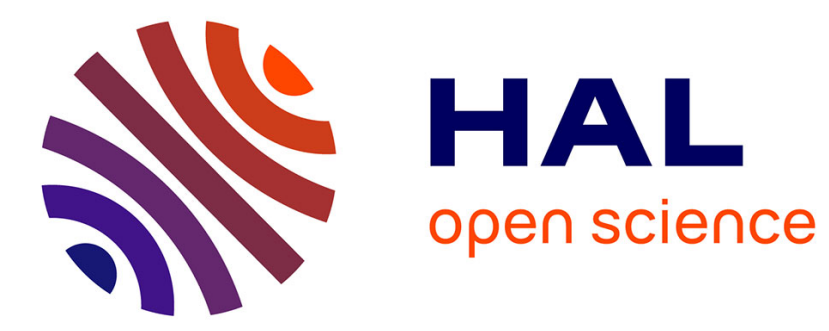

\title{
Concise derivation of scattering function from channel entropy maximization
}

François-Xavier Socheleau, Christophe Laot, Jean-Michel Passerieux

\section{To cite this version:}

François-Xavier Socheleau, Christophe Laot, Jean-Michel Passerieux. Concise derivation of scattering function from channel entropy maximization. IEEE Transactions on Communications, 2010, 58 (11), pp.3098 - 3103. 10.1109/TCOMM.2010.091310.090247 . hal-00565754

\section{HAL Id: hal-00565754 https://hal.science/hal-00565754}

Submitted on 10 Jun 2021

HAL is a multi-disciplinary open access archive for the deposit and dissemination of scientific research documents, whether they are published or not. The documents may come from teaching and research institutions in France or abroad, or from public or private research centers.
L'archive ouverte pluridisciplinaire HAL, est destinée au dépôt et à la diffusion de documents scientifiques de niveau recherche, publiés ou non, émanant des établissements d'enseignement et de recherche français ou étrangers, des laboratoires publics ou privés. 


\title{
Concise Derivation of Scattering Function from Channel Entropy Maximization
}

François-Xavier Socheleau*‡, Student Member, IEEE, Christophe Laot*, Member, IEEE, and Jean-Michel Passerieux $\ddagger$

\begin{abstract}
In order to provide a concise time-varying SISO channel model, the principle of maximum entropy is applied to scattering function derivation. The resulting model is driven by few parameters that are expressed as moments such as the channel average power or the Doppler spread. Physical interpretations of the model outputs are discussed. In particular, it is shown that common Doppler spectra such as the flat or the Jakes spectrum fit well into the maximum entropy framework. The Matlab code corresponding to the proposed model is available at http://perso.telecom-bretagne.eu/fxsocheleau/software.
\end{abstract}

\section{Index Terms}

Scattering function, entropy, channel model, propagation, underwater acoustic communications

\section{INTRODUCTION}

The scattering function (SF) fully characterizes the second-order statistics of a random, linear timevarying process that satisfies the wide-sense stationary uncorrelated scattering (WSSUS) assumption [1]. In the context of mobile communication, the SF expresses the time-frequency selectivity of a fading channel so that its knowledge is of practical interest in numerous applications such as code design [2], multicarrier system design [3], channel simulation [4] or channel capacity analysis [5]. The performance of these applications depends on the chosen SF. This SF is usually either provided by theoretical considerations on the physical environment [6] or by direct estimation during ongoing data transmission [7], or results from real-world channel sounding experiments [8]. These various approaches mainly rely on a given state of channel knowledge and a priori assumptions.

\footnotetext{
*Institut Telecom; Telecom Bretagne; UMR CNRS 3192 Lab-STICC, Université européenne de Bretagne, Technopôle Brest Iroise-CS 83818, 29238 Brest Cedex, France. Email: \{fx.socheleau, christophe.laot\}@telecom-bretagne.eu

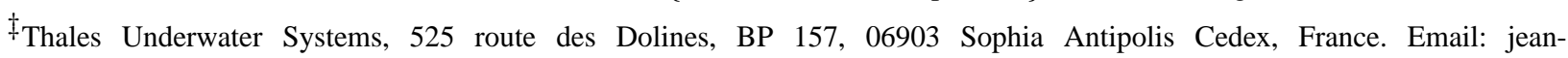
michel.passerieux@fr.thalesgroup.com.
} 
In some applications where few data are available, intuitive assumptions are often preponderant to fulfill the lack of knowledge on the channel properties. A relevant illustration is underwater acoustic communication channel modeling where, unlike radio channels, there is no consensus on the statistical characterization of both the probability distribution and the power spectral density of the fading process [9]. Such ad-hoc model construction may result in inconsistencies. In our context, consistent modeling is defined as the requirement that independent modelers with the same state of channel knowledge must obtain identical models. The consistency argument is a key element in statistical inference [10]. In [11], Debbah et al. address the question of consistent channel modeling in light of Bayesian probability theory and the principle of maximum entropy introduced in [12] by Jaynes. Classically, the maximum entropy problem consists in deriving a probability density function (pdf) from a finite set of expectations. Maximizing entropy is shown to be the only way to provide a consistent model that is maximally noncommittal with respect to unavailable information [13]. Choosing the model with the greatest entropy therefore avoids the arbitrary introduction of information that is not known.

As emphasized in [12], maximum entropy modeling is not meant to represent the physical reality but rather a state of knowledge of this reality. A crucial task is then to determine the quantity and the kind of information that the model needs in order to characterize the environment to model with enough accuracy. Ideally, modelers seek to constraint their model with parameters that are very informative and easy to know or to estimate for a given environment. This desideratum is mainly justified by the will to provide an accurate channel model that does not require difficult-to-obtain environmental parameters.

The model presented in [11] is very general and considers doubly dispersive channels in a multipleinput multiple-output (MIMO) context. Its derivation relies on spatial considerations and requires some knowledge on the angles of departure (resp. arrival) between the scatterers and the transmitting (resp. receiving) antenna. While spatial considerations suit the MIMO context very well, it may no be the case for single-input single-output (SISO) channels. In practice, the distribution of the angles of departure or arrival can hardly be estimated and is rarely known a priori in a SISO scenario, expect for rich scattering environments (dense urban or indoor communications) where an isotropic radiation is assumed.

As an alternative to [11] in a SISO context, we here propose a channel model where the time fluctuations are derived from the knowledge of some moments of the SF rather than from a given knowledge on the distribution of the angles of departure or arrival. In the maximum entropy framework, SF moments prove to be relevant parameters to constraint SISO models since they are (i) very informative, in the sense that only a few moments are needed to get an accurate model (ii) easy to estimate when real data are available and (iii) easy to physically interpret so that their order of magnitude is usually known a priori 
for a given environment. A typical example addressed in this paper is the derivation of SF with the only knowledge of the channel average power and Doppler spread. It is shown that this approach leads to a concise model where the channel behavior can be expressed through few variables.

The paper is organized as follows. Section II reviews some basic characterizations of WSSUS channels. After recalling some results of [11] to model the channel pdf, the principle of maximum entropy is applied to SF derivation in section III. In section IV, we show some outputs provided by the resulting channel model and give a physical interpretation of their entropy. Finally, conclusions are given in section V. Additionally, the Matlab code corresponding to the model is available at http://perso.telecombretagne.eu/fxsocheleau/software.

\section{WSSUS CHANNEL MODEL}

A propagation channel can be usually modeled as a linear random time-varying system defined by its impulse response $h(\tau, t)$ or equivalently by its (delay-Doppler) spreading function $S(\tau, \nu)$ such that the input $x(t)$ and the output $y(t)$ of this system satisfy

$$
y(t)=\int_{-\infty}^{\infty} h(\tau, t) x(t-\tau) d \tau=\int_{-\infty}^{\infty} \int_{-\infty}^{\infty} S(\tau, \nu) x(t-\tau) e^{2 i \pi \nu t} d \tau d \nu .
$$

$S(\tau, \nu)$ characterizes the attenuation and scatterer reflectivity associated with paths of delay $\tau$ and Doppler $\nu$. It verifies $S(\tau, \nu)=\int_{-\infty}^{\infty} h(\tau, t) e^{-2 i \pi \nu t} d t$.

Generally, a statistical characterization of the channel is preferred to a deterministic description since it provides a more concise channel representation. In this case, the impulse response is modeled as a twodimensional random process. The second-order statistics of the channel then depends on four variables. A significant simplification is obtained by invoking the wide-sense stationary uncorrelated scattering (WSSUS) assumption [1]. For WSSUS channels, the correlation is reduced from four to two dimensions so that scatterers with different delay or different Doppler are uncorrelated, i.e.,

$$
\mathbb{E}\left[S(\tau, \nu) S^{*}\left(\tau^{\prime}, \nu^{\prime}\right)\right]=C(\tau, \nu) \delta\left(\tau-\tau^{\prime}\right) \delta\left(\nu-\nu^{\prime}\right)
$$

where $C(\tau, \nu)$ is the so-called scattering function defined as

$$
C(\tau, \nu)=\mathbb{E}\left[|S(\tau, \nu)|^{2}\right]=\int_{-\infty}^{\infty} \mathbb{E}\left[h(\tau, t) h^{*}(\tau, t+\Delta t)\right] e^{-2 i \pi \nu \Delta t} d \Delta t .
$$

In a baseband equivalent discrete-time setting, the channel input-output relation satisfies

$$
y[k]=\sum_{l=0}^{L-1} h_{l}[k] x[k-l]
$$


where $x[k], y[k]$, and $h_{l}[k]$ are sampled versions of $x(t), y(t)$, and $h(\tau, t)$, with sampling frequency larger than the system bandwidth. $L$ denotes the total number of channel taps. The channel is assumed to be a multi-variate random process expressed as $H(k)=\left[h_{0}[k], \cdots, h_{L-1}[k]\right]$.

In discrete-time, the scattering function becomes

$$
C_{l}(\nu)=\sum_{u=-\infty}^{+\infty} \mathbb{E}\left[h_{l}[k] h_{l}^{*}[k+u]\right] e^{-2 i \pi \nu u} .
$$

For $l$ fixed to $l=l_{0}, C_{l_{0}}(\nu)$ corresponds to the power spectral density or Doppler power spectrum of the random process $h_{l_{0}}[k]$. Note that $C_{l}(\nu)$ is discrete in delay but continuous in Doppler.

\section{MAXIMUM ENTROPY MODELING}

\section{A. Gaussian channel model}

The vast majority of models assume that the channel impulse response is a Gaussian process. The assumption usually relies on the physical argument that for a fixed delay the received signal is the sum of the contribution of a large number of scattered reflections. Instead of being assumed, Gaussianity can be formally justified in light of inductive inference and the principle of maximum entropy [11].

For instance, in the case where the only known information about the channel is that its total energy is finite and equal to $\boldsymbol{\sigma}^{2}$, the channel probability density function $P(H)$ can be obtained by maximizing the entropy

$$
-\int \log P(H) d P(H)
$$

under the constraint that

$$
\int d P(H)=1 \text { and } \int \sum_{l=0}^{L-1}\left|h_{l}\right|^{2} d P(H)=\sigma^{2} .
$$

Note that the index $k$ is voluntary omitted when there are no references to time-related properties. This maximization problem can be solved using the method of Lagrange multipliers

$$
\mathrm{L}(P)=-\int \log P(H) d P(H)+\alpha\left(1-\int d P(H)\right)+\lambda\left(\boldsymbol{\sigma}^{2}-\int \sum_{l=0}^{L-1}\left|h_{l}\right|^{2} d P(H)\right)
$$

and setting the functional derivative of $L(P)$ to 0

$$
\frac{\delta \mathrm{L}(P)}{\delta P}=-\log P(H)-1-\alpha-\lambda \sum_{l=0}^{L-1}\left|h_{l}\right|^{2}=0 .
$$

Solving the system of equations (7) and (9) then leads to i.i.d and Gaussian distributed channel taps [11], i.e.,

$$
P(H)=\frac{1}{\left(\pi \boldsymbol{\sigma}^{2} / L\right)^{L}} \prod_{l=0}^{L-1} e^{-\frac{L\left|h_{l}\right|^{2}}{\boldsymbol{\sigma}^{2}}}
$$


As opposed to classical channel model derivation, Gaussianity as well as taps independence were not prior assumptions but a consequence of the only constraint of finite energy.

Each time a new information on the channel is available, the model of Eq. (10) can be refined. For instance, in many applications the channel power-delay profile is known so that each tap $l$ has a power given by $\mathbb{E}\left[\left|h_{l}\right|^{2}\right]=\sigma_{l}^{2}+\left|s_{l}\right|^{2}$ where $s_{l}=\mathbb{E}\left[h_{l}\right]$ is the expression of a potential specular component (that can be null). In this case, the channel pdf that maximizes the entropy becomes

$$
P(H)=\prod_{l=0}^{L-1} \frac{1}{\left(\pi \sigma_{l}^{2}\right)} e^{-\frac{\left|h_{l}-s_{l}\right|^{2}}{\sigma_{l}^{2}}} .
$$

This result indicates that when the channel knowledge is limited to its first and second order statistics, the less arbitrary model to implement according to the principle of maximum entropy is the Gaussian model. A Gaussian pdf induces Rice or Rayleigh fading depending on whether $s_{l}$ is null or not.

\section{B. Scattering function derivation}

From the result of Eq. (11), it can be deduced that the joint entropy of the multivariate random process $H$ can be expressed as the sum of the entropy of each process $h_{l}$. Thus, maximizing the entropy of $H$ is equivalent to maximizing the entropy of the different $h_{l}$ independently. Therefore, the SF that maximizes the entropy is obtained by independent derivation of the Doppler spectrum of each channel tap. For this reason and for the sake of readability the index $l$ is voluntary omitted throughout this subsection.

The dynamic of a stationary Gaussian process is fully characterized by its second order statistics or "color" that is expressed under the stationary assumption by its power spectral density or Doppler power spectrum $C(\nu)$ (see Eq. (5)). Thanks to Kolmogorov, the entropy rate $\overline{\mathrm{h}}$ of a discrete-time Gaussian processes $h[k]$ can be expressed as a function of this power spectral density so that [14]

$$
\overline{\mathrm{h}}=\frac{1}{2} \log (2 \pi e)+\frac{1}{2} \int_{-1 / 2}^{1 / 2} \log C(\nu) d \nu .
$$

The process $h[k]$ is implicitly assumed to be sampled at a period that verifies Shannon's theorem such that $C(\nu)$ has a bounded normalized support $\left[\nu_{\min }, \nu_{\max }\right]$ with $-1 / 2 \leq \nu_{\min } \leq \nu \leq \nu_{\max } \leq 1 / 2$.

To be consistent with the principle of maximum entropy as articulated previously, our objective is to find, for each tap, the $C(\nu)$ that maximizes the entropy rate $\overline{\mathrm{h}}$ subject to some constraints that translates our state of knowledge of the channel time fluctuations. In general, this knowledge can be expressed for each tap as a set of spectral moments $\mu_{p}$ of known functions $m_{p}(\nu), p \in \mathcal{P}$ where $\mathcal{P}$ denotes the set of constraint orders, i.e.,

$$
\mu_{p}=\int_{\nu_{\min }}^{\nu_{\max }} m_{p}(\nu) C(\nu) d \nu
$$


In maximum entropy spectral analysis [15], it is classically assumed that at least $N$ equally-spaced points of the autocorrelation function are known. In this case, $\mu_{p}$ corresponds to the autocorrelation function $\mu_{p}=\mathbb{E}\left[h[k] h^{*}[k+p]\right]$ that can also be expressed as trigonometric spectral moments such that $m_{p}(\nu)=e^{2 i \pi p \nu}$ and $\mathcal{P}=\{0,1, \cdots, N-1\}$. Such representation of the channel may not be adapted to concise modeling since it requires the knowledge of $N$ correlation points. A shortcut toward concise modeling could be to choose $N$ small but this may not be a good option since it may not characterize the channel with enough accuracy. An alternative approach is to express our state of knowledge of the channel time fluctuations in terms of geometrical moments such that $m_{p}(\nu)=\nu^{p}$. Such moments have the main advantage of being easy to physically interpret so that their order of magnitude is usually known for a given environment. Typically, $\mu_{0}$ is the total average power of $h[k], \mu_{1}$ is the barycenter of $C(\nu)$ (that can be seen as an indicator of spectral symmetry) and $\mu_{2}$ expresses the root-mean-square (rms) Doppler spread $D_{s}$ of $h[k]$ such that $D_{s}=\sqrt{\mu_{2} / \mu_{0}-\left(\mu_{1} / \mu_{0}\right)^{2}}$.

Therefore, from Eqs (12) and (13), the analytical expression of the SF is obtained by solving, for each tap, the following optimization problem

$$
\begin{array}{cl}
\max _{C(\nu)} & \int_{\nu_{\min }}^{\nu_{\max }} \log C(\nu) d \nu, \\
\text { subject to } & \int_{\nu_{\min }}^{\nu_{\max }} \nu^{p} C(\nu) d \nu=\mu_{p}, \forall p \in \mathcal{P} .
\end{array}
$$

As articulated in the previous subsection, the solution is provided thanks to the Lagrange multipliers

$$
\mathrm{L}(C)=\int \log C(\nu) d \nu-\sum_{p \in \mathcal{P}} \lambda_{p}\left(\mu_{p}-\int \nu^{p} C(\nu) d \nu\right) .
$$

By differentiating $\mathrm{L}(C)$ with respect to $C$, we get

$$
C(\nu)=\frac{1}{\sum_{p \in \mathcal{P}} \lambda_{p} \nu^{p}}
$$

The multipliers $\lambda_{p}$ are then found by solving the set of non-linear equations

$$
\int_{\nu_{\min }}^{\nu_{\max }} \frac{\nu^{q}}{\sum_{p \in \mathcal{P}} \lambda_{p} \nu^{p}} d \nu=\mu_{q}, \forall q \in \mathcal{P}
$$

Depending on the set $\mathcal{P}$, an analytical solution of such system of equations is in general not straightforward to obtain. Therefore, to facilitate the computation of the multipliers $\lambda_{p}$, a numerical approach such as the one presented in appendix $\mathrm{A}$ is advocated.

\section{ILLUSTRATIONS}

Through three case studies, we analyze in this section the behavior of the Doppler spectrum $C(\nu)$ as well as the entropy of the associated process when it is subjected to various constraints. In particular, we 
analyze the maximum entropy model constrained by the spectral moments from a "spatial" perspective. An example of practical application of the proposed model is also discussed. It shows, for instance, that second-order moments can be very informative. As we focus on concise modeling, the channel time fluctuations are here only constrained by the spectral moment $\mu_{2}$ that is homogeneous to the square of the Doppler spread. Unless otherwise stated, in all the following examples, $\nu_{\min }=-1 / 2, \nu_{\max }=1 / 2$ and $\mu_{0}$ is set to 1 (unitary power).

\section{A. Flat Doppler Spectrum}

The first question to answer when using the proposed method is: "What is the time-varying channel model maximally non-committal with respect to unavailable information when almost nothing is known about its time fluctuations?". The term "almost" expresses the idea that even if $\nu_{\min }, \nu_{\max }$ and the $\mu_{p}, p \geq 1$ are unknown, it is always possible to bound the channel dynamic by physical considerations. Typically, $\nu_{\min }$ and $\nu_{\max }$ can be upper-bounded by $\nu_{\text {limit }}$ that is obtained from the knowledge of the transmission carrier frequency $f_{c}$, the speed of wave propagation $c$ and the maximum relative speed between the transmitter and the receiver $v_{\text {limit }}$ (max. car speed in urban communications, max. boat or AUV speed in underwater communications etc.), i.e $\nu_{\text {limit }}=v_{\text {limit }} f_{c} / c$. Moreover, to maximize the channel entropy, the inphase and quadrature components of each tap have to be uncorrelated which is equivalent to considering symmetrical Doppler power spectrum, i.e. $\nu_{\min }=-\nu_{\max }$. The principle of maximum entropy then attributes a uniform distribution to $\nu_{\max }$ over $\left[0, \nu_{\text {limit }}\right]$. Once $\nu_{\max }$ is randomly drawn and given that nothing more is known about the channel dynamic, $C(\nu)$ is given, according to Eq. (16), by

$$
C(\nu)=\frac{1}{2 \nu_{\max }},-\nu_{\max } \leq \nu \leq \nu_{\max }
$$

This flat Doppler spectrum proved to be the one that maximizes the entropy when there is (almost) no prior on the channel dynamic. This is in total agreement with the physical interpretation of a flat Doppler spectrum. As shown in [6], this spectrum corresponds to a 3-D isotropic scattering environment where the angles of arrival are uniformly distributed in both the elevation and the azimuth plane. We recall that the uniform distribution represents the state of maximum ignorance according to [12].

\section{B. Jakes Doppler Spectrum}

The Jakes Doppler spectrum is widely used in mobile wireless propagation and is expressed for $\nu_{\min }=$ $-1 / 2, \nu_{\max }=1 / 2$ and $\mu_{0}=1$ as

$$
C(\nu)=\frac{1}{\pi / 2 \sqrt{1-(2 \nu)^{2}}}
$$


The Jakes spectrum is based on the assumption that the angles of arrival of the radio-waves at reception are uniformly distributed over $[0,2 \pi]$ in the azimuth plane. Thanks to this uniform distribution and even if Eq. (19) cannot be analytically expressed as in Eq. (16), this spectrum should well fit into the maximum entropy framework. To validate this hypothesis, the maximum entropy model is constrained by $\mu_{2}=1 / 8$, the rms Doppler spread of Eq. (19) being $1 / \sqrt{8}$. Theoretically, there is an infinite number of spectrum with $\mu_{2}=1 / 8$ but as shown in Figure 1 , the one that maximizes the entropy is very close to the Jakes spectrum. The maximum entropy spectrum gets even closer to Jakes' when a fourth-order constraint is applied to our model.

\section{Entropy rate vs Doppler spread}

Figure 2 presents the evolution of the entropy rate as a function of $\mu_{2}$. Because the Doppler spread $\sqrt{\mu_{2}}$ cannot exceed the Doppler width equal to $\left(\nu_{\max }-\nu_{\min }\right) / 2=1 / 2, \mu_{2}$ is upper-bounded by $1 / 4$. The first observation on this figure is that the overall maximum entropy is obtained for $\mu_{2}=1 / 12$ which corresponds, in agreement with subsection IV-A, to a flat Doppler spectrum. Moreover, for limit cases where $\mu_{2}=0$ or $1 / 4$, the entropy tends to $-\infty$. These cases correspond to a Doppler spectrum that can be expressed as Dirac delta functions. According to [14], the minimum mean-squared error (MMSE) of the best estimator of a sample of the process given the infinite past is

$$
\operatorname{MMSE}=\frac{1}{2 \pi e} 2^{2 \overline{\mathrm{h}}}
$$

so that an entropy equaling $-\infty$ leads to a totally predictable process. Another strong deduction that can be made from this equation is that when there is no noise, the performance of causal adaptive channel estimation is only dependent on the channel entropy and not directly on the Doppler spread or the shape of the Doppler spectrum. Channels with a different SF but with the same entropy rate can theoretically be estimated with the same performance. Figure 3 shows two Doppler spectra with the same entropy of 1.8 but with a different shape and a different Doppler spread.

\section{Example of a practical application}

The proposed model can be of practical interest in various applications such as those listed in the introduction. For instance, from the only knowledge of some moments of the SF, it is possible to generate channel impulse response realizations that can be useful to help the design of communication systems ${ }^{1}$.

\footnotetext{
${ }^{1}$ The channel realizations can be generated from the SF using sum-of-sinusoids methods or by Gaussian processes filtering [4].
} 
This is particularly relevant in domains like underwater acoustics where the research community agrees on the order of magnitude of some moments such as the Doppler spread but where there is yet no consensus on the full statistical characterization of the channel [9]. Figure 4-(a) shows an example of a real underwater acoustic channel probed in the Atlantic ocean at $17.5 \mathrm{kHz}$ and over a bandwidth of 3 kHz. Figure 4-(b) displays a realization of the maximum entropy simulator trying to "mimic" this real channel. The simulator is constrained with the values of $\mu_{0}, \mu_{2}$ and $\nu_{\max }$ measured on the real data recorded at sea. To assess the accuracy of the simulator, we compare in Figure 5 the level crossing rate (LCR) of the original channel with the one resulting from the maximum entropy model. The LCR is defined as the rate at which the channel envelope crosses a specified level $\rho$ in the positive slope [4]. This statistics, that depends on the power spectral density of the fading process, is a way of characterizing the severity of the fading over the time and is commonly employed to design interleavers and error-correcting codes. It can be seen that there is a good match between the statistics of the two channels. Note that the channel impulse response probed at sea is a single realization of the underlying random process so that its fading statistics are estimated with a large variance whereas the results of the maximum entropy model are averaged over 100 realizations. Complementary statistics (not shown here) such as average fade durations or bit error rates have been measured and also indicate that bounding the parameters to the second-order moments (i.e. $\sup \{\mathcal{P}\}=2$ ) usually convey enough information to make the model accurate.

\section{CONCLUSiON}

Throughout this paper, we have shown that the maximum entropy principle proves to be a relevant framework for a concise and consistent derivation of the scattering function. An exhaustive knowledge of the propagation phenomena is not required to obtain a channel model. Fading statistics measurements indicate that limiting this knowledge to the channel average power and Doppler spread can lead to accurate models.

\section{REFERENCES}

[1] P. A. Bello, "Characterization of randomly time-variant linear channels," IEEE Trans. Commun. Systems, vol. 11, no. 4, pp. 360-393, 1963.

[2] H. Bolcskei, R. Koetter, and S. Mallik, "Coding and modulation for underspread fading channels," in IEEE Int. Symp. Information Theory, 2002.

[3] P. Jung and G. Wunder, “The WSSUS Pulse Design Problem in Multicarrier Transmission,” IEEE Trans. Commun., vol. 55, no. 10, 2007.

[4] M. Patzold, Mobile fading channels, Wiley, 2002.

[5] G. Durisi, U.G. Schuster, H. Bolcskei, and S. Shamai, "Noncoherent Capacity of Underspread Fading Channels," IEEE Trans. Inf. Theory, vol. 56, no. 1, 2010. 
[6] R. H. Clarke and W. L. Khoo, "3-D Mobile Radio Channel Statistics," IEEE Trans. Veh. Technol., vol. 46, no. 3 , 1997.

[7] H. Artes, G. Matz, and F. Hlawatsch, "Unbiased Scattering Function Estimators for Underspread Channels and Extension to Data-Driven Operation,” IEEE Trans. Signal Process., vol. 52, no. 5, pp. 254-264, 2004.

[8] COST 207, "Digital and Land Mobile Radio Communications," Office for Official Publications of the European Communities, Final report, Luxembourg, 1989.

[9] M.Stojanovic and J.Preisig, "Underwater Acoustic Communication Channels: Propagation Models and Statistical Characterization,” IEEE Commun. Mag., pp. 84-89, Jan. 2009.

[10] E. T. Jaynes, Probability Theory: The Logic of Science, Cambridge Univ. Press, 2003.

[11] M. Debbah and R. R. Muller, "MIMO Channel Modeling and the Principle of Maximum Entropy," IEEE Trans. Inf. Theory, vol. 51, no. 5, pp. 1667-1690, 2005.

[12] E.T. Jaynes, “Information Theory and Statistical Mechanics,” Physical review, vol. 106, no. 4, pp. 620-630, 1957.

[13] J. Shore and R. Johnson, "Axiomatic Derivation of the Principle of Maximum Entropy and the Principle of Minimum Cross-Entropy," IEEE Trans. Inf. Theory, vol. 26, no. 1, pp. 26-37, 1980.

[14] T. Cover and J. Thomas, Elements of Information Theory, Wiley, 1991.

[15] J. P. Burg, Maximum Entropy Spectral Analysis, Ph.D. thesis, Department of Geophysics, Stanford University, 1975.

[16] A. M. Djafari, “A Matlab Program to Calculate the Maximum Entropy Distributions,” http://djafari.free.fr/pdf/me91_1.pdf.

[17] P. Bello, "Some Techniques for the Instantaneous Real-Time Measurement of Multipath and Doppler Spread," IEEE Trans. Commun. Tech., vol. 13, no. 3, pp. 285-292, 1965.

[18] J. Holtzman and A. Sampath, “Adaptive Averaging Methodology for Handoffs in Cellular Systems," IEEE Trans. Veh. Technol., vol. 44, no. 1, 1995 .

\section{APPENDIX A}

\section{NUMERICAL SOLUTION OF EQUATION (17)}

To obtain the lagrange multipliers $\lambda_{p}$ of Eq. (17), a simple gradient based method as the one presented in [16] is suggested. The vector $\boldsymbol{\Lambda}=\left[\lambda_{p}\right]_{p \in \mathcal{P}}$ is found by solving the system

$$
G_{q}(\boldsymbol{\Lambda})=\int_{\nu_{\min }}^{\nu_{\max }} \frac{\nu^{q}}{\sum_{p \in \mathcal{P}} \lambda_{p} \nu^{p}} d \nu=\mu_{q}, q \in \mathcal{P} .
$$

This equation is solved iteratively by first developing the $G_{q}(\boldsymbol{\Lambda})$ in Taylor's series around an initial vector $\Lambda^{0}$.

$$
G_{q}(\boldsymbol{\Lambda}) \approx G_{q}\left(\boldsymbol{\Lambda}^{0}\right)+\left(\boldsymbol{\Lambda}-\boldsymbol{\Lambda}^{0}\right)^{T} \operatorname{grad}\left(G_{q}(\boldsymbol{\Lambda})\right)_{\left(\boldsymbol{\Lambda}=\boldsymbol{\Lambda}^{0}\right)} .
$$

If $\epsilon$ and $v$ are defined as

$$
\epsilon=\boldsymbol{\Lambda}-\boldsymbol{\Lambda}^{0}, v=\left[\mu_{q}-G_{q}\left(\boldsymbol{\Lambda}^{0}\right)\right]_{q \in \mathcal{P}}^{T} \text { and } \Gamma=\left(\gamma_{q n}\right)=\left(\frac{\partial G_{q}(\boldsymbol{\Lambda})}{\partial \lambda_{n}}\right)_{\left(\boldsymbol{\Lambda}=\boldsymbol{\Lambda}^{0}\right)}
$$

then $\Gamma \epsilon=v$. Note that the matrix $\Gamma$ is symmetric so that

$$
\gamma_{q n}=\gamma_{n q}=-\int_{\nu_{\min }}^{\nu_{\max }} \frac{\nu^{q+n}}{\left(\sum_{p \in \mathcal{P}} \lambda_{p} \nu^{p}\right)^{2}} d \nu .
$$

At each iteration, $\epsilon$ is computed and a new $\Lambda^{0}$ that verifies $\Lambda^{0}=\Lambda-\epsilon$ is set. The iterations continue until $\epsilon$ is small enough. 


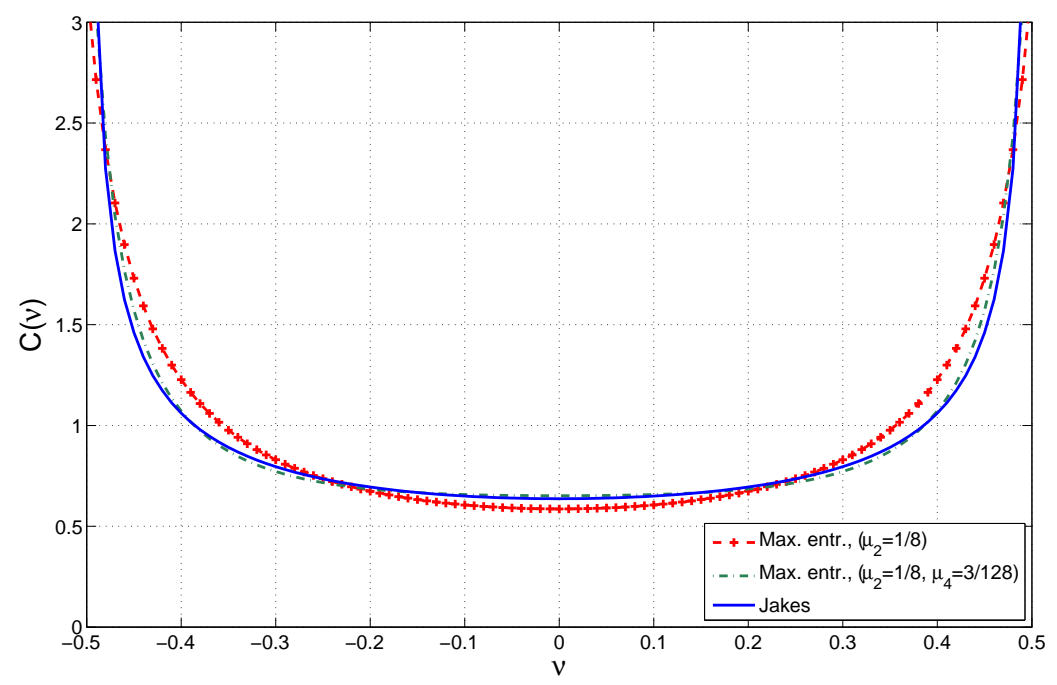

Fig. 1. Jakes Doppler spectrum approximated by entropy maximization. 


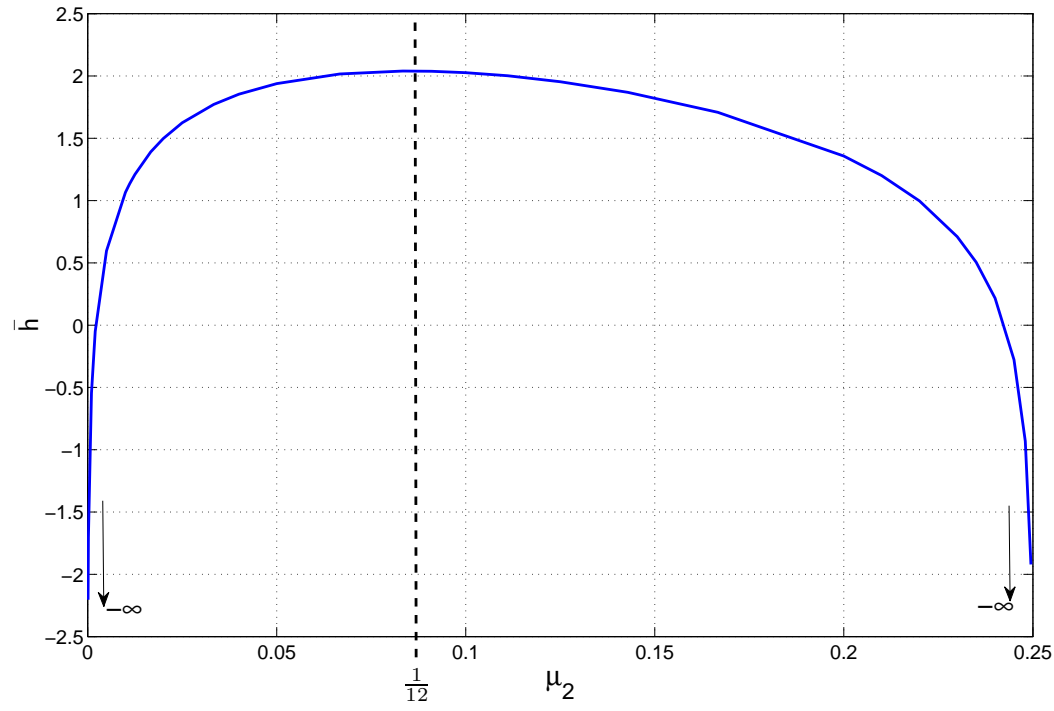

Fig. 2. Channel entropy as a function of the second-order spectral moment $\mu_{2}$.

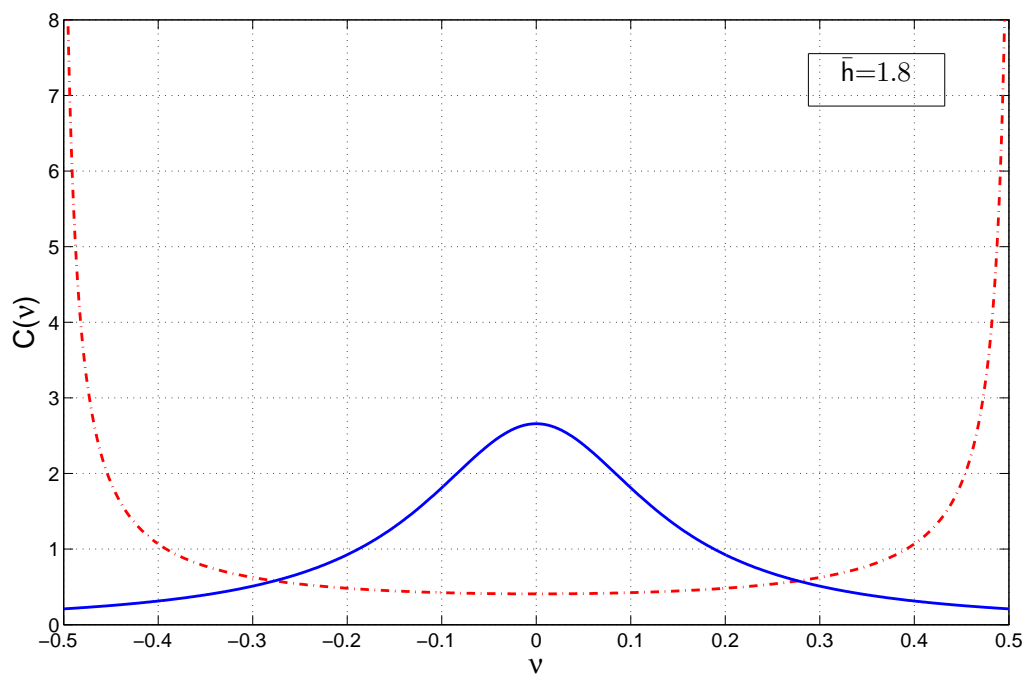

Fig. 3. Illustration of two Doppler spectra with a different Doppler spread resulting in Gaussian processes with the same entropy. $\mu_{2}=0.0355$ for the plain line spectrum and $\mu_{2}=0.1531$ for the dash-dot line spectrum. 


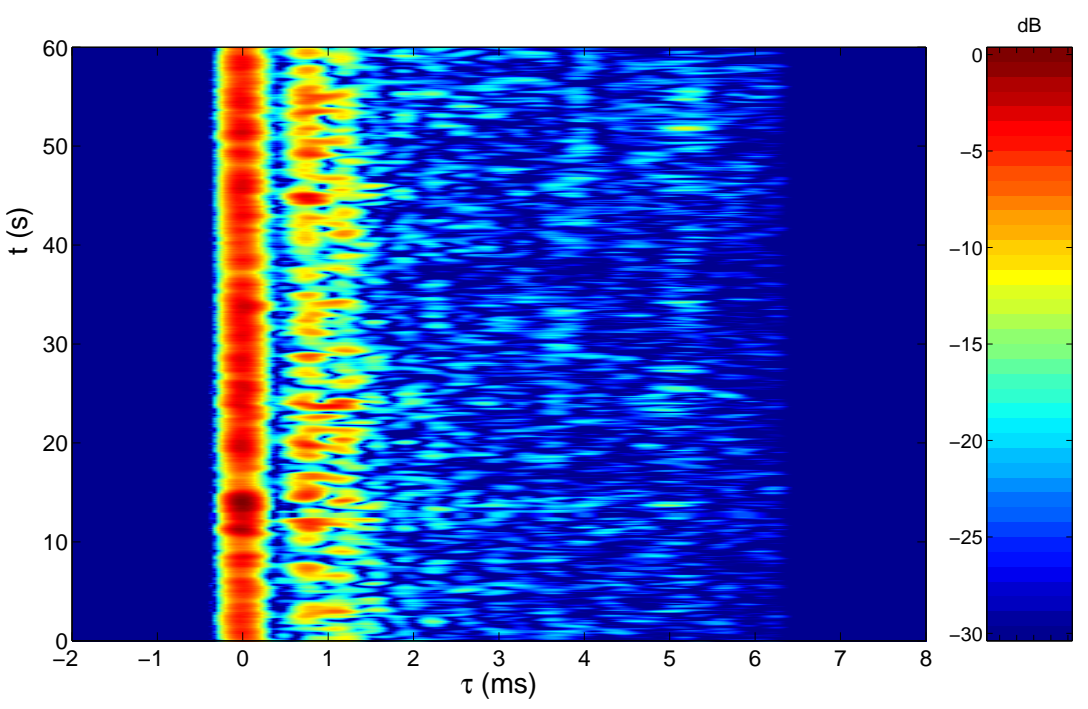

(a)

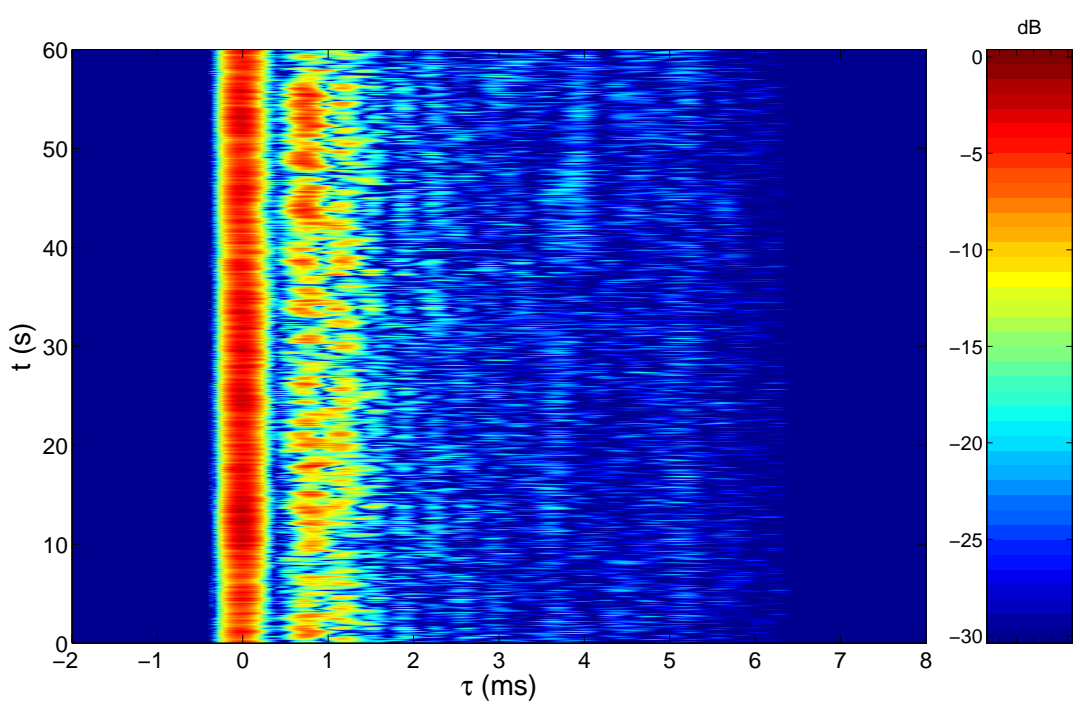

(b)

Fig. 4. Comparison between a real underwater acoustic channel probed in the Atlantic ocean and a realization of its equivalent maximum entropy model. (a) Real probed channel, (b) Maximum entropy channel. The rms Doppler spread of each tap is estimated using the algorithm presented in [17] and ranges from 0.7 to $3.8 \mathrm{~Hz}$. The maximum Doppler frequency is measured using the method detailed in [18] and is equal to $6.5 \mathrm{~Hz}$. 


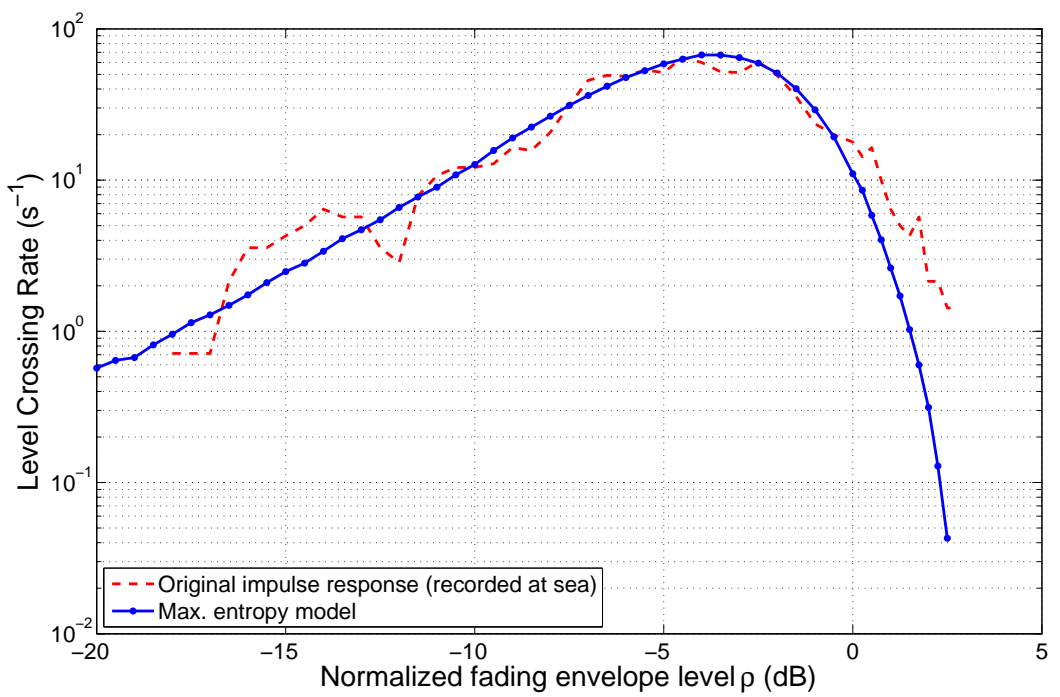

Fig. 5. Fading statistics comparison between a real underwater acoustic channel probed in the Atlantic ocean and a realization of its equivalent maximum entropy model. 